\title{
New findings and interpretation on atmospheric adsorption induced instability in microcrystalline silicon films
}

\author{
Tae-Hyeon Lim, Hyuk-Ryeol Park \\ Department of Physics, Mokpo National University, Muan, Jeonnam, Republic of Korea, 534-729
}

Email address:

thlim@mokpo.ac.kr (Tae-Hyeon L.),hrpark@mokpo.ac.kr (Hyuk-Ryeol P.)

\section{To cite this article:}

Tae-Hyeon Lim, Hyuk-Ryeol Park. New Findings and Interpretation on Atmospheric Adsorption Induced Instability in Microcrystalline Silicon Films. International Journal of Materials Science and Applications. Vol. 3, No. 3, 2014, pp.100-105.

doi: $10.11648 /$ j.ijmsa.20140303.15

\begin{abstract}
In this investigation, we added some new findings and interpretations on the behaviors of both electrical conductivities and optical absorption spectra upon adsorption of atmospheric molecules in microcrystalline silicon films ( $\mu \mathrm{c}-\mathrm{Si}: \mathrm{H})$. The photo conductivity followed similar decay-recover processes upon air exposure-thermal anneal cycles as did the dark conductivity. The dark and photo conductivities decay according to a stretched-exponential function with time upon air exposure. The absorption spectra showed a reduced defect absorption for the films at air exposed state compared with those of at thermal annealed state. There is a one-to-one correspondence between atmospheric adsorption in $\mu \mathrm{c}-\mathrm{Si}: \mathrm{H}$ and bias-stress induced instabilities in a-Si:H thin film transistors(TFTs).
\end{abstract}

Keywords: Microcrystalline Silicon, Atmospheric Adsorption Instability, Stretched-Exponential Decay, Band-Bending

\section{Introduction}

One of the common instabilities in $\mu \mathrm{c}-\mathrm{Si}: \mathrm{H}$ films is a decrease of dark conductivity $\sigma_{d}$ caused by adsorption of atmospheric gases. For some $\mu \mathrm{c}-\mathrm{Si}: \mathrm{H}$ films, $\sigma_{d}$ decreases up to several orders of magnitude during the storage of films in the air, of which initial value may be restored by annealing in vacuum at around $200^{\circ} \mathrm{C}$. The annealed value may remain stable when the film is kept in vacuum, whereas it changes again when put back into the air [1-8].

Even though a wide range of probing techniques and samples has been used in previous investigations, this complex and intriguing instability problem still remains to be solved. Along with $\sigma_{d}$, the steady-state photo conductivity $\sigma_{p h}$ measurements have served as a basic tool to characterize a-Si:H and $\mu \mathrm{c}-\mathrm{Si}: \mathrm{H}$ films. The behaviors of $\sigma_{p h}$ during decay-recover processes upon air exposure-thermal anneal cycles have not been confirmed. Also, the dependence of $\sigma_{d}$ on the exposure time in atmospheric gases has not been defined mathematically. Furthermore, there have been very little concerns about the behaviors of the sub-gap optical absorption spectra upon this instability. The present study simultaneously examined the behaviors of $\sigma_{d}, \sigma_{p h}$ and the optical absorption spectra during annealing and air exposure.
The purpose of this study is to reveal whether there exist any correlations among $\sigma_{d}, \sigma_{p h}, \mathrm{CPM}$ spectra upon atmospheric adsorption. Secondly, we want to define the dependence of $\sigma_{d}$ on the exposure time mathematically. Thirdly, we will try to interpret an atmospheric aging phenomena of $\mu \mathrm{c}-\mathrm{Si}: \mathrm{H}$ on the basis of the charge trapping model which was used in a-Si:H TFTs to explain the threshold voltage shift upon gate-bias stresses.

\section{Experiments}

Intrinsic $\mu \mathrm{c}-\mathrm{Si}: \mathrm{H}$ films were deposited by $13.56 \mathrm{MHz}$ PECVD system at LG Display Company, at a gas pressure of 1 5 Torr, under a silane concentration $\mathrm{SC}$ ([ $\left.\mathrm{SiH}_{4}\right] /$ $\left.\left[\mathrm{SiH}_{4}\right]+\left[\mathrm{H}_{2}\right]\right)$ of $1 \sim 11 \%$, and an RF power of $800 \sim 2000$ $\mathrm{W}$, onto glass substrate of $20 \mathrm{~cm} \times 20 \mathrm{~cm}$ area maintained at $250 \sim 300{ }^{\circ} \mathrm{C}$. Crystalline volume fraction $X_{C}$ was estimated from a Raman scattering experiment. The film thickness measured with a stylus profiler (Dektak, BRUKER) was between $0.8 \sim 1.5 \mu \mathrm{m}$ A-Si:H layer of $100 \AA$ was deposited first to avoid peeing-off films from smooth glass substrate. The coplanar electrodes $10 \mathrm{~mm}$ in length and $200 \mu \mathrm{m}$ in gap separation were deposited for the electrical conductivity measurements using a Keithley 
electrometer and a data acquisition system. The temperature dependence of conductivity measurement and annealing were done consecutively by heating the film from room temperature to above $200^{\circ} \mathrm{C}$ (heating), staying thereat for 1-2 hours (annealing), and coming back to room temperature (cooling), in vacuum. Adsorbing of atmospheric molecules was performed by keeping the films in the dark laboratory atmosphere at room temperature. Sub-gap optical absorption spectra were measured by CPM at room temperature in vacuum and calibrated with conventional optical transmission spectra. In CPM measurements, the film is illuminated from the coplanar electrode side with chopped $(13 \mathrm{~Hz})$ monochromatic light. The resulting photocurrent was converted to a voltage by a preamplifier and the voltage signal was fed into a lock-in amplifier. The incident light intensity was measured with $\mathrm{Si}$ and Ge calibrated photodiodes. All measurements were performed in vacuum cryostat under a pressure of $1 \sim 2 \times 10^{-5}$ mbar. Our investigation was focused for the highly crystalline samples at low SC yielding high $X_{C}$ values of above $50 \%$. It was previously revealed that highly crystalline films with porous, large grain size and columns separated by deep crack show a significant adsorption effect [3].

\section{Experimental Results}

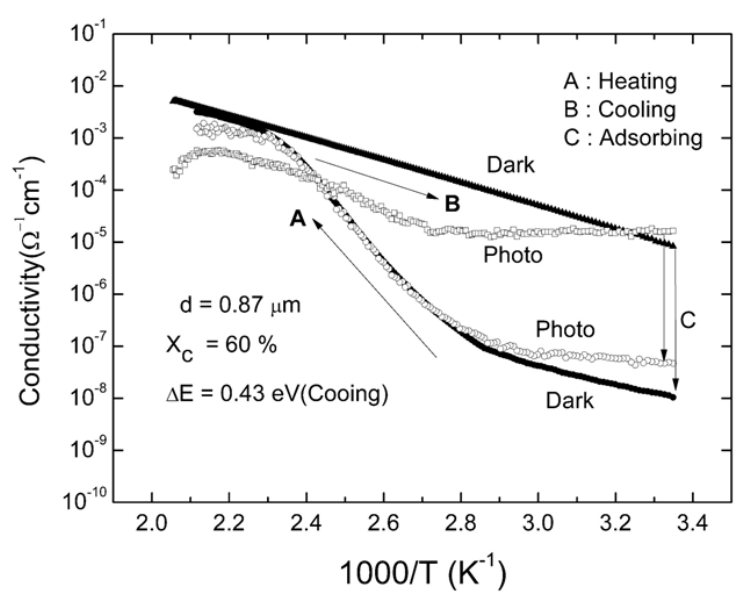

Figure 1. The representative behavior of $\sigma_{d}(T)$ and $\sigma_{p h}(T)$ changes during heating-annealing-cooling-adsorbing cycle for a $\mu \mathrm{c}-\mathrm{Si}: \mathrm{H}$ film. Adsorbing run was conducted over two weeks by keeping the film in the dark atmosphere at room temperature. $\sigma_{p h}$ was measured with a band-pass $(650 \pm 50 \mathrm{~nm})$ filtered halogen lamp light at an intensity of 50 $\mathrm{mW} / \mathrm{cm}^{2}$.

Fig. 1 is the behavior of $\sigma_{d}$ vs. $1000 / T$ during heating-annealing-cooling-adsorbing cycle for $\mu \mathrm{c}-\mathrm{Si}: \mathrm{H}$ film with $X_{C}$ values of $60 \%$. In previous reports, same type of figures were suggested as a typical evidence of aging phenomenon upon atmospheric adsorption in $\mu \mathrm{c}-\mathrm{Si}: \mathrm{H}$ films $[1,3,5,6]$. Adsorbing run at fixed room temperature has led to a decrease in $\sigma_{d}$ of about three orders of magnitude. $\sigma_{d}(T)$ at cooling run after annealing shows a thermally activated type of conduction with an activation energy $\Delta E$ of $0.43 \mathrm{eV} . \sigma_{d}(T)$ at heating run after adsorbing run shows also a thermally activated type of conduction but with downward kink or double activation energies. If we apply the single activation energy conduction for $\sigma_{d}(T)$ at heating run, then $\Delta E$ is given to be about 1.0 eV.Consequently, adsorption of atmospheric gases effectively induced a movement of the dark Fermi level $E_{F}$ from $0.43 \mathrm{eV}$ to $1.0 \mathrm{eV}$ below conduction band edge.

By atmospheric adsorption, the dark conductivity can be either decreased or increased. It has been postulated that the decrease (increase) is related to the electron acceptor (donor) such as oxygen (water) molecules adsorbed on the surface of the film, within cracks or at grain boundaries[1-8]. The adsorption ofelectron acceptor (donor) molecules will create upward (downward) band-bending and thereby establish depletion (accumulation) layer at the surface, which will result in a decrease (increase) in conductivity. This is equivalent to a movement of the Fermi level away from (close to) the conduction band.

What a newly reported in this study is the behavior of $\begin{array}{llll}\sigma_{p h} & \text { vs. } & 1000 / T & \text { during }\end{array}$ heating-annealing-cooling-adsorbing cycle. A significant correlation is noted between $\sigma_{d}(T)$ and $\sigma_{p h}(T)$ behaviors upon atmospheric aging. For $\sigma_{p h}$, after a movement of $E_{F}$ near middle of the band gap, the number of recombination centers for conduction electron would be increased, thereby the lifetime of conduction electrons and eventually $\sigma_{p h}$ was decreased.

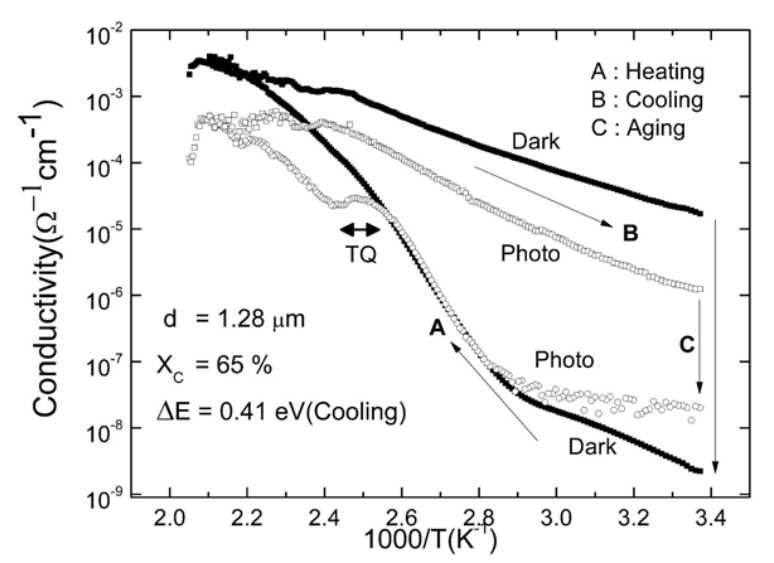

Figure 2. The behavior of $\sigma_{d}(T)$ and $\sigma_{p h}(T)$ changes during heating-cooling-aging cycle for $\mu \mathrm{c}-\mathrm{Si}$ :H film with $X_{c}$ of $65 \%$ and $\Delta E$ of $0.41 \mathrm{eV}$.Thermal quenching range is designated as $T Q$ on $\sigma_{p h}(T)$ curve.

For $\mu \mathrm{c}-\mathrm{Si}: \mathrm{H}$ films with a significant aging effect, we also observed that $\sigma_{p h}$ vs. $1000 / T$ showed diverse behaviors, of which $\sigma_{d}$ vs. $1000 / T$ was basically the same. Fig.2 is another $\sigma_{p h}$ vs. $1000 / T$ result for a film with a thickness of $1.28 \mu \mathrm{m}$. In Fig.2, the noticeable one is the occurrence of thermal quenching effect. This suggests that there are at least two sets of states, differing each other in their relative magnitude of capture cross section for electrons and holes [9]. One set is the recombination center which has about equal capture cross section for electrons and holes, while the other is the hole trap which has the vanishingly small capture 
cross section for electrons. We believe that there exist various sets of states on surface and interface in highly crystalline films that show a significant aging effect. The detailed aspects of these states are currently not understood. The interaction between these states and free carriers in conduction and valence band could introduce diverse behaviors in their $\sigma_{p h}(T)$ vs. $1000 / T$ spectra.

We examined the time rate of $\sigma_{d}$ and $\sigma_{p h}$ decay upon air exposure for a $\mu \mathrm{c}-\mathrm{Si}: \mathrm{H}$ film. For the first time, the observed curves were fitted with a stretched-exponential(SE) function such as given below

$$
\sigma(t)=\sigma_{s S}-\left(\sigma_{s S}-\sigma_{0}\right) \exp \left[-(t / \tau)^{\beta}\right]
$$

where $\sigma_{0}$ and $\sigma_{s s}$ are the annealed-state and the fully adsorbed-state value of conductivity, $\beta$ is a stretching factor and $\tau$ is a time constant for the decay. The results are shown in Fig. 3, where the values of $\beta$ are 0.40 for $\sigma_{d}$ and 0.42 for $\sigma_{p h}$, and $\tau$ are $80 \mathrm{~min}$ for $\sigma_{d}$ and $250 \mathrm{~min}$ for $\sigma_{p h}$. In mathematical context, a linear rate equation $d n / d t=$ $-k n$ with a rate-constant $k$ which has either a distribution of its value or a power-law time dependence gives a solution of SE function [9]. Here the rate equation stands for a relaxation process of a specific property $n$ of a physical system that evolves toward equilibrium after the sudden enforcement of a perturbation, and the characteristic rate constant $k$ account for a dispersive effect involved in this relaxation process. So far, several microscopic models for this dispersion factor have been suggested. They include percolation, hierarchical relaxation of constraints, multi-polar interaction transitions, a continuous-time random walk, and a multiple trapping-detrapping mechanism[10].

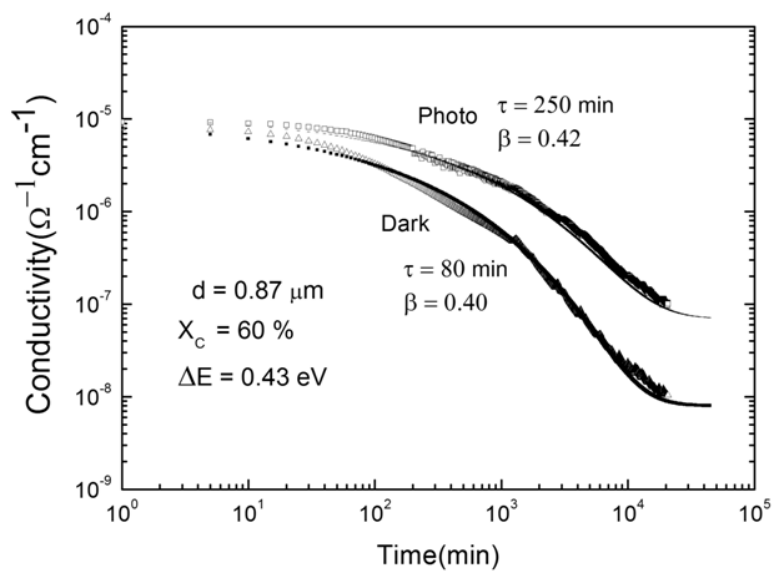

Figure 3. The time rate of both $\sigma_{d}$ and $\sigma_{p h}$ decay upon air exposure at room temperature for a $\mu \mathrm{c}-\mathrm{Si}$ :H film which is same with one used for Fig. 1. The symbols are data points and the lines are the result of stretched exponential fittings using $\tau$ and $\beta$ parameter values as are designated on the figure.

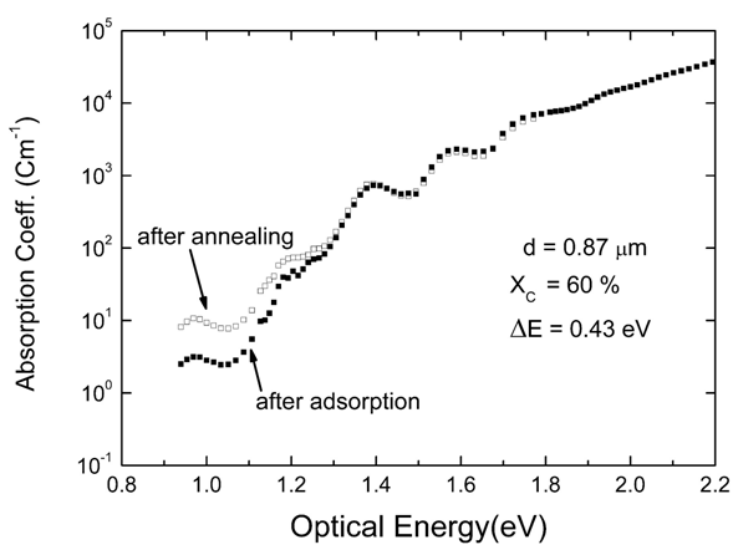

Figure 4. CPM optical absorption spectra for a $\mu c-$ Si:Hfilm with a thickness of $0.87 \mu \mathrm{m}$. The spectra were measured after atmospheric adsorption or after thermal annealing, respectively. CPM was run within high vacuumchamber to prevent any atmospheric adsorption during measurement.

Fig.4 is the CPM optical absorption spectra measured after annealing and adsorption. The spectra show the general features of intrinsic $\mu \mathrm{c}-\mathrm{Si}: \mathrm{H}$ films, which had a larger optical absorption (in comparison with c-Si) at above $1.8 \mathrm{eV}$ due to an amorphous silicon tissue and another increase at $1.3 \sim 1.8 \mathrm{eV}$ (in comparison with a-Si:H) due to a crystalline grain and grain boundary. What a noticeable in Fig. 4 is a reduction of spectrum below $1.3 \mathrm{eV}$ after adsorption compared with one after annealing. Fig. 5 is another result for a film with a thickness of $1.28 \mu \mathrm{m}$. A similar result was reported by Gunes et al.[8]. They used the dual beam photoconductivity method to measure sub-gap optical absorption for $\mu \mathrm{c}-\mathrm{Si}: \mathrm{H}$ films with thickness between $200 \mathrm{~nm}$ and $350 \mathrm{~nm}$. In the meantime, we have used the single beam photoconductivity method without dc bias-light and films much thicker than theirs. Bias-light and the thinness of films could introduce a complication into this aging phenomenon. Fig. 6 is the spectral response of photocurrent, measured under constant light intensity for the film used in Fig. 5. The amplitude of spectrum measured after annealing is larger than that of measured after adsorption by about two orders. Here, raw spectra were normalized at $1.8 \mathrm{eV}$ to a constant valuein order to see the relative changes in the sub- and above- band gap energies. Again a reduction of amplitude after adsorption compared with one after annealing is observed in the range of sub-band gap energies. The reduction in above-band gap energies could be explained as due to an increased surface recombination rate which is caused by Fermi level shift after adsorption. What a noticeable in Fig. 6 is the small peaks that are appeared at about $3.1 \mathrm{eV}$. We interpret these peaks as the optical transition from $L_{3}^{\prime}$ to $L_{1}$ critical point in crystalline $\mathrm{Si}$ [11]. This result may be a reflection of high crystallinity of the films used in this study. 


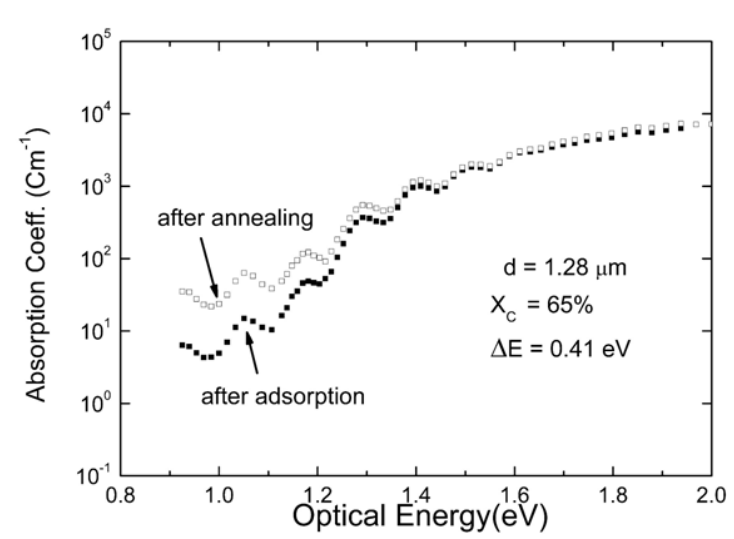

Figure 5. CPM optical absorption spectra for a $\mu c-S i: H$ film with a thickness of $1.28 \mu \mathrm{m}$.

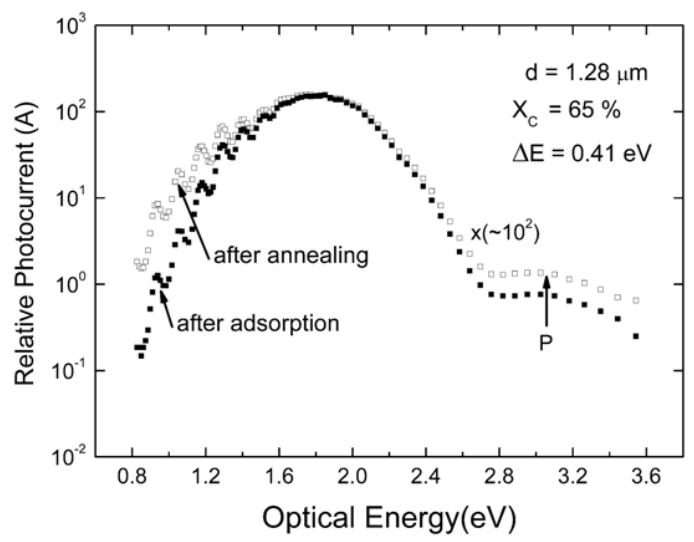

Figure 6. The spectral response of photocurrent, measured under constant light intensity for the film used in Fig. 5. P in the figuredenotes the possible peaks corresponding to the optical transition from $L_{3}{ }^{\prime}$ to $L_{1}$ critical point in crystalline Si..

In thin-film transistor (TFT), a band-bending between gate and source-drain channel is established by applying voltage to gate electrode. Positive (Negative) bias makes downward (upward) band-bending. Furthermore, the prolonged application of gate bias which is called as the bias-stress can also induce a built-in band-bending after clearing bias-stress. Positive (Negative) bias-stress makes upward (downward)built-in band-bending due to the trapped electrons (holes) within gate insulator. Previously, we applied CPM to measure optical absorption spectra of a-Si:H films in TFT structure and the behavior of CPM spectra upon band-bending between gate and source-drain channel were investigated[12,13]. The observed spectra showed a reduced defect absorption, compared with that under flat-band condition, when band-bending (either upward or downward) is formed at a-Si:H/a-SiN:H interface. Upon this argument, the reduction in CPM spectra after adsorption in Fig. 5 and Fig. 6 can be interpreted as due to a band-bending at surface of $\mu \mathrm{c}-\mathrm{Si}: \mathrm{H}$ film upon adsorption of atmospheric molecules. During atmospheric adsorption, electron acceptor molecule such as oxygen will be adsorbed onto the exposed area between coplanar electrodes of $\mu \mathrm{c}-\mathrm{Si}: \mathrm{H}$ film and thereby establish an upward band-bending from bulk to surface[1,3]. After thermal annealing and thereby desorption of adsorbed molecules, an upward band-bending is cleared and CPM spectrum will be returned to its flat-band value.

\section{Discussions}

The charge-trapping effect is well known instability in a-Si:HTFFs. In n-channel a-Si:H TFT, under positive gate bias, the downward band-bending is established at $\mathrm{a}-\mathrm{Si}: \mathrm{H} / \mathrm{a}-\mathrm{SiN}_{\mathrm{x}}: \mathrm{H}$ interface and thereby channel electrons which leak into the gate $\mathrm{a}-\mathrm{SiN}_{\mathrm{x}}: \mathrm{H}$ are captured by traps therein. After clearing gate bias, the trapped electrons in the $\mathrm{a}-\mathrm{SiN}_{\mathrm{x}}: \mathrm{H}$ generate upward band-bending at $\mathrm{a}-\mathrm{Si}: \mathrm{H} / \mathrm{a}-\mathrm{SiN}_{\mathrm{x}}: \mathrm{H}$ interface. As a result, threshold voltage shifts positively, drain current decreases up to several orders, and also sub-gap optical absorption spectra decreases significantly. The threshold voltage shift caused by charge-trapping was shown to follow SE function[14-18]. It was also shown that SE function fits to the normalized drain current changes under positive bias-stress at fixed temperature[19].

There seems to be a one-to-one correspondence among the observed phenomena from atmospheric adsorption of $\mu \mathrm{c}-\mathrm{Si} \mathrm{H}$ and bias-stress induced instabilities of a-Si:H TFTs. The positive Fermi level shift and the dark conductivity decrease in atmospheric adsorption of $\mu \mathrm{c}-\mathrm{Si}: \mathrm{H}$ may correspond to the positive threshold voltage shift and the drain current decrease in positive bias-stress induced instabilities in a-Si:H TFTs, respectively. Furthermore, the same resultswere observed for the CPM spectra in $\mu \mathrm{c}-\mathrm{Si}: \mathrm{H}$ and a-Si:H TFTs with a built-in upward band-bending[8,12,13].Furthermore, dark conductivity increase and negative Fermi level shift in atmospheric adsorption of $\mu \mathrm{c}-\mathrm{Si}: \mathrm{H}$ may correspond to the drain current increase and the negative threshold voltage shift in negative bias-stress induced instabilities in a-Si:H TFTs. In TFTs, Negative bias-stress makes downward built-in band-bending due to the trapped holes within gate insulator. It has been generally accepted that $\sigma_{d}$ increase in $\mu \mathrm{c}-\mathrm{Si}: \mathrm{H}$ is related with the physical adsorption of electron donor molecules such as water molecules during exposure to air[1-6]. The established adsorbate layer will then create anaccumulation layer or downward band-bending at and adjacent to the surface. This is equivalent to a movement of the Fermi level close to the conduction band, which is seen by the increase of $\sigma_{d}$.

In charge-trapping model for a-Si:H TFT, if one assumes that all electrons that enter the insulator are trapped, the threshold voltage shift depends strictly on the process of electron tunneling into the gate insulator, and has a logarithmic time dependence[15]. On the other hand, if most electrons that enter the gate insulator are not trapped and continue to the gate electrode, the threshold voltage shift can be shown to follow SE function [16-18]. A dispersive effect involved in this relaxation process is the distribution of capture cross section of traps for electron trapping within gate nitride [17]. $\beta$ is a measure of the 
distribution width, with a value between 0 and 1 . The $\beta$ decreases as the distribution broadens, and $\beta=1$ implies a single trap cross section. In the meantime, a dispersion factor for relaxation of atmospheric adsorption remains to be clarified. In atmospheric adsorption in $\mu \mathrm{c}-\mathrm{Si}: \mathrm{H}$ films, the process is a physical adsorption which is derived through Van de Waals-type forces. Since it is non-dissociative, the distribution of activation energy for adsorption is not expected. The atmospheric aging effect depends on the microstructure of the films[3,6,7]. The interaction of a molecule with a given surface will be dependent upon the physical and chemical environment of surface. They may include local atomic configurations and any existing adsorbed species, such as surface impurities or simply pre-adsorbed molecules of the same type, etc. The difference of these factors from site to site could yield the distribution width of capture cross section for adsorption. As the number of surface sites occupied by adsorbates increases with time, so does the probability that it will visit a site with a low value of capture cross section against attraction of the adsorbate. When the distribution is broad, the rate-constant for adsorption will reflect these low rate-constant values and the adsorption will become slower at longer times to produce a SE-type relaxation rather than a normal exponential decay.

\section{Conclusion}

For $\mu \mathrm{c}-\mathrm{Si}: \mathrm{H}$ films with high crystalline volume fraction, we have found that their photo conductivities also show a similar aging effect upon air exposure as does their dark conductivities. The time rate of both dark and photo conductivity decay upon air exposure at room temperature can be fitted to the stretched exponential function. The reduction of CPM optical absorption spectrum at air exposed state was found. The Fermi level shift, the stretched exponential decay of conductivities and sub-gap absorption spectra reductions upon atmospheric aging can be interpreted by employing the charge trapping model on the bias-stress induced instability in a-Si:H TFTs.

\section{Acknowledgements}

This paper was supported by Research Funds of Mokpo National University in 2013.

\section{References}

[1] S. Veprek, Z. Iqbal, R. O. Kuhne, P. Capezzuto, F-A. Sarott and J. K. Gimzewski, "Properties of microcrystalline silicon IV. Electrical conductivity, electron spin resonance and the effect of gas adsorption", J. Phys. C: Solid State Phys,.vol. $16,6241(1983)$.

[2] V. Smirnov, S. Reynolds, C. Main, F. Finger, R. Carius, "Aging effects in microcrystalline silicon films studied by transient photoconductivity", J. Non-Cryst. Solids, vol.338, 421 (2004).
[3] F. Finger, R. Carius, T. Dylla, S. Klein, S. Okur, M. Gunes, "Instability phenomena in microcrystalline silicon films", J. of Optoelectronics and Advanced Materials, vol. 7(1), 83 (2005).

[4] R. Reynolds, V. Smirnov, F. Finger, R. Carius, "Transport and instabilities in microcrystalline silicon ", J. of Optoelectronics and Advanced Materials, vol. 7(1), 91 (2005).

[5] M. Gunes, R.E. Jonson, S. O. Kasap, F. Finger, and A. Lambertz, "The effect of aging on the dark conductivity and $1 / \mathrm{f}$ noise in hydrogenated microcrystalline silicon thin films", Phys. Status Solidi., vol. C7 No. 3-4, 658 (2010).

[6] G. Yilmaz, E. Turan, M. Gunes, V. Smirnov, F. Finger, and R Bruggemann, " Instability effects in hydrogenated microcrystalline silicon thin films", Phys. Status Solidi., vol. C7 No. 3-4, 700 (2010).

[7] R. Bruggemann and N. Souffi, "Metastable dark and photoconductive properties of microcrystalline silicon", J. Non-Cryst. Solids, vol. 352, 1079 (2006).

[8] M. Günes, H. Cansever, G. Yilmaz, V. Smirnov, F. Finger, R Bruggemann, "Metastability effects in hydrogenated microcrystalline silicon thin films investigated by the dual beam photoconductivity method", J. Non-Cryst. Solids, vol.358, 2074 (2012).

[9] A. Rose, "Concepts in Photoconductivity and allied problems", edited by R. E. Marshak (Interscience Publishers, New York·London, 1963) p. 51

[10] J. Kakalios, R. A. Street, and W. B. Jackson, "Stretched-exponential relaxation arising from dispersive diffusion of hydrogen in amorphous silicon", Phys. Rev. Lett., vol.59(9), 1037 (1987).

[11] L. Van Hove, "The Occurrence of Singularities in the Elastic Frequency Distribution of a Crystal," Phys. Rev., vol. 89, 1189 (1953).

[12] Hyuk-Ryeol Park, Dong-Sun Oh, Jin Jang, and Hoe-Sup Soh, "Optical absorption spectra of a-Si:H film in TFT structure",Solid. St. Commun., vol. 97(1), 45 (1996).

[13] Hyuk-Ryeol Park, Dong-Sun Oh, Jin Jang, "Changes in bulk defect density of hydrogenated amorphous silicon by bias stress in thin film transistor structure", Appl. Phys. Lett., vol. 68(22), 3135 (1996).

[14] F. R. Libsch J. Kanicki, “ Bias-stress-induced stretched-exponential time dependence of charge injection and trapping in amorphous thin-film transistors", Appl. Phys. Lett., vol.62(11), 1286 (1993).

[15] M. J. Powell, "Charge Trapping Instabilities in Amorphous Silicon-Silicon Nitride ThinFilm Transistors," Applied Physics Letters, vol. 43, 597 (1983).

[16] J. Triska, J. F. Conley, R. Presley, and J. F. Wager, "Bias stress stability of zinc-tin-oxidethin-film transistors with $\mathrm{Al} 2 \mathrm{O} 3$ gate dielectrics", Journal of Vacuum Science \&Technology B,vol.28, C5il-C5i6, (2010).

[17] S. Zafar, A. Callegari, E. Gusev, and M. V. Fischetti, "Charge trapping related thresholdvoltage instabilities in high permittivity gate dielectric stacks," J. Appl.Phys., vol.93, 9298 (2003). 
[18] A. V. Gelatos and J. Kanicki, "Bias Stress-Induced Instabilities in Amorphous-Silicon Nitride Hydrogenated Amorphous-Silicon Structures - Is the Carrier-Induced Defect Creation Model Correct," Appl. Phys. Lett., vol. 57. 1197 (1990).
[19] C. C. Shih, Y. S. Lee, K. L. Fang, C. H. Chen, and F. Y. Gan, "A current estimation method for bias-temperature stress of a-Si TFT device", IEEE Transactions on Device and Materials Reliability, vol. 7, 347 (2007). 